\title{
ESTIMATING THE HEDGE RATIOS
}

\author{
Mária Bohdalová ${ }^{1}$, Michal Greguš ${ }^{2}$
}

\begin{abstract}
This paper examines the problem of hedging portfolio returns. Many practitioners and academicians endeavor to solve the problem of how to calculate the optimal hedge ratio accurately. In this paper we compare estimates of the hedge ratio from a classical approach of a linear quantile regression, based on selected quantiles as medians, with that of a non-linear quantile regression. To estimate the hedge ratios, we have used a calibrated Student $t$ distribution for the marginal densities and a Student $t$ copula of the portfolio returns using a maximum likelihood estimation. We created two portfolios of the assets, one for equal weight and another for optimal weight in respect of minimal risk. Our findings show that an assumption of Student $t$ marginal leads to a better estimation of the hedge ratio.
\end{abstract}

JEL Classification Numbers: C21, C40, G11 DOI: http://dx.doi.org/10.12955/cbup.v4.874

Keywords: quantile regression, hedge ratio, copula.

\section{Introduction}

Contemporary financial markets are characterized by their instability due to many crisis periods. Academic literature is a rich source of trading strategies that are oriented to eliminate the risk of losses. Hedging is one of them. Hedging strategies have also been examined using models that incorporate tail dependence structure between the investment of interest and the hedging instrument (Lien, Shrestha, \& Wu, 2016). Conventional method for the optimal hedge ratios estimate uses the regression method. This approach is mainly based on the expected relation between investment of interest and the hedging instrument returns, which neglect the relationships between these two returns at different quantiles. This paper uses the concept of a quantile hedge ratio. We use a quantile regression method in estimating these quantile hedge ratios using copula. The quantile hedge ratio allows investment of interest and the hedging instrument returns to have different relationships at different quantiles. Consequently, a higher investment return may require a different hedge ratio from a lower investment return (Brooks, Olan, \& Persand, 2002; Alexander, 2008). When the relationship between investment and hedging instrument returns does vary across quantiles, the quantile hedge ratio can provide better hedging efficiency than the hedge ratio obtained by OLS.

The purpose of this paper is to determine the hedge ratios of two portfolios - Equally Weighted (EW) and Minimum Variance (MV) portfolio when the investor decides to reduce his risk. For this purpose

1. we calibrate both portfolios and the hedge instrument by an appropriate distribution using historical prices time series,

2. we compare the symmetric and asymmetric hedge ratio:

- using simple linear regression based on OLS

- using linear quantile regression

- using non-linear quantile regressions based on Normal and Student $t$ copula.

The paper is organized as follows. The next section presents the methodology used in estimating the optimal hedge ratios. Section 3 describes the analyzed data and section 4 discusses empirical results. The conclusion concludes the paper.

\section{Methodology}

Let $S_{t}$ and $F_{t}$ represent investment and hedged instrument prices at time $t$. Then, returns on investment and hedged positions can be defined as $\Delta s_{t}=\ln \left(S_{t}\right)-\ln \left(S_{t-1}\right)$ and $\Delta f_{t}=\ln \left(F_{t}\right)-\ln \left(F_{t-1}\right)$ respectively. The return on the hedged portfolio $R_{H t}$ is given by (Lien et al., 2016):

$$
R_{H t}=\Delta s_{t}-\mathrm{H} \Delta f_{t},
$$

\footnotetext{
${ }^{1}$ Mária Bohdalová, Comenius University in Bratislava, Faculty of Management, Slovak Republic, Maria.Bohdalova@fm.uniba.sk

${ }^{2}$ Michal Greguš, Comenius University in Bratislava, Faculty of Management, Slovak Republic, Michal.gregus@fm.uniba.sk
} 
where $H$ is the so-called hedge ratio.

The $M V$ hedge ratio $H_{M V}$ is obtained by minimizing the variance of $R_{H}$ with respect to $H$ and is given by (Lien et al., 2016):

$$
H_{M V}=\frac{\operatorname{cov}\left(\Delta s_{t}, \Delta f_{t}\right)}{\operatorname{Var}\left(\Delta f_{t}\right)}
$$

In the conventional approach, $M V$ hedge ratio is represented as the coefficient in a regression of investment returns on hedging returns:

$$
\Delta s_{t}=\alpha+\beta \Delta f_{t}+e_{t}
$$

where the estimate of the $M V$ hedge ratio, $H_{M V}$, is given by the estimate of the slope coefficient $\beta$.

The OLS method has been frequently criticized because this technique considers only symmetric linear relationship between variables and cannot provide a distinction between dependence during up and down markets or between large and small stock price movements (Aymen \& Mongi, 2016). Quantile regression technique (developed by Koenker \& Bassett in 1978) is an extension of the traditional least squares estimation of the conditional mean to a compilation of models for different conditional quantile functions. Classical linear regression model that describes the dependence of $Y$ on $X$ assumes the variables are bivariate normal. If $X$ and $Y$ do not have a bivariate normal distribution, then it is needed to describe the conditional distribution $F(Y \mid X)$. Quantile regression gives tools to describe the conditional distribution of the dependent variable using its quantiles (Alexander, 2008). Quantile regression expresses the conditional quantiles of $Y$ given $X$ based on an arbitrary joint distribution while it is assumed the errors of the quantile regression are i.i.d with specific error distribution function $F_{\varepsilon}$.

Let $q$ be a quantile of the error determined by $F_{\varepsilon}^{-1}(q), q \in(0,1)$ and simultaneously $q$ denote the conditional quantile of the dependent variable $Y$, which is found from the inverse of $F(Y \mid X)$, by $F^{-1}(q \mid X)$. Now, we take conditional quantile $q$ of a simple linear regression model

$$
Y=\alpha+\beta X+\varepsilon
$$

and we get a simple linear quantile regression model (Alexander, 2008):

$$
F^{-1}(q \mid X)=\alpha+\beta X+F_{\varepsilon}^{-1}(q \mid X) .
$$

The aim of the simple quantile regression is to estimate the regression line parameters $\alpha$ and $\beta$ based on a paired sample of $X$ and $Y$, while quantile regression line passes through a quantile of the points. Quantile regression lines for different values of $q$ are not parallel. Dependence of $Y$ based on explanatory variable $X$ could be constant when the values of $(\alpha, \beta)$ are not changed for different values of $q$, or monotonically increasing (decreasing) when $(\alpha, \beta)$ increases (decreases) with the value of $q$ and symmetric (asymmetric) when the value of $q$ is similar (dissimilar) for lower and upper quantiles (Aymen \& Mongi, 2016). The coefficients $(\alpha, \beta)$ for a given $q$ are estimated by minimizing the weighted sum of the absolute errors as

$$
\min _{\alpha, \beta} \sum_{t=1}^{T}\left(q-1_{Y_{t} \leq \alpha+\beta X_{t}}\right)\left|Y_{t}-\left(\alpha+\beta X_{t}\right)\right|,
$$

where

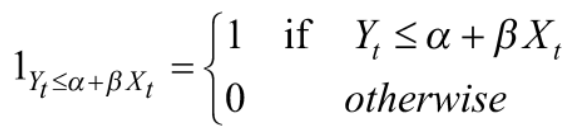

The solution $\left(\hat{\alpha}_{q}, \hat{\beta}_{q}\right)$ that minimizes the quantile loss function (3) satisfies

$$
\hat{F}^{-1}(q \mid X)=\hat{\alpha}_{q}+\hat{\beta}_{q} X+F_{\varepsilon}^{-1}(q \mid X),
$$


where $\hat{F}^{-1}(q \mid X)$ is the sample estimate of the conditional $q$-quantile. We have used a numerical solution of equation (6) in Wolfram Mathematica software to estimate quantile regression coefficients $\left(\hat{\alpha}_{q}, \hat{\beta}_{q}\right)$.

Copula quantile approach replaces the linear model in (6) by the $q$ quantile curve of a copula. (Bouyé $\&$ Salomon, 2002). This enables us to model nonlinear relationships (Alexander, 2008).

Let the marginal distributions of $X$ and $Y$ be $F(X)$ and $G(Y)$. They are specified by their marginal parameters $\alpha=\left\{\alpha_{X}, \alpha_{Y}\right\}$, which we have already estimated using maximum likelihood method. Let $F(X$, $\left.\alpha_{X}\right)$ and $G\left(Y, \alpha_{Y}\right)$. We then specify some functional form for a bivariate copula, which depends on certain parameters $\theta$ (the normal bivariate copula has one parameter, the correlation coefficient $\rho$ and the bivariate Student $t$ copula has two parameters, the degrees of freedom $v$ and the correlation $\rho$ ). Then the $q$ quantile regression curve is $Y_{t}=Q_{q}\left(X_{t}, q ; \hat{\alpha}_{q}, \hat{\theta}_{q}\right)$, where $\left\{\hat{\alpha}_{q}, \hat{\theta}_{q}\right\}$ solves the optimization problem

$$
\min _{\alpha, \theta} \sum_{t=1}^{T}\left(q-1_{Y_{t} \leq Q_{q}\left(X_{t}, q ; \alpha, \theta\right)}\right)\left(Y_{t}-Q_{q}\left(X_{t}, q ; \alpha, \theta\right)\right)
$$

The explicit function $Y=Y_{t}$ of the normal copula quantile curves can be written as (Alexander, 2008):

$$
Y=G^{-1}\left[\Phi\left(\rho \Phi^{-1}(F(X))+\sqrt{1-\rho^{2}} \Phi^{-1}(q)\right)\right]
$$

and Student $t$ copula quantile curves has form

$$
Y=G^{-1}\left[t_{v}\left(\rho t_{v}^{-1}(F(X))+\sqrt{\left(1-\rho^{2}\right)(v+1)^{-1}\left(v+t_{v}^{-1}(F(X))^{2}\right)} t_{v+1}^{-1}(q)\right)\right] .
$$

\section{Data}

This paper analyses the risk of investing in a portfolio created from these three stocks: Bayer (BAYN), Siemens (SIE) and Volkswagen Group (VOW). We have chosen the DAX index as the hedged instrument.

We have estimated the optimal hedge ratio for the risk of our portfolios. We have created two portfolios - equally weighted (EW) and mean-variance (MV) optimal portfolio. MV portfolio is composed from $24.153 \%$ of BAYN, $69.269 \%$ SIE and only $6.578 \%$ VOW.

Closing spot prices for both portfolios and the DAX index $\left(P_{t}\right.$ measured in time $\left.t\right)$ were rebased to 100 and the fluctuation (measured in terms of logarithmic return) was computed. The analyzed period was from September, 27, 2010 to June, 28, 2016, which comprised 1,500 trading days, or T=1,499 logarithmic returns (log returns are obtained by formula: $r_{t}=\ln P_{t} / \ln P_{t-1}, t=1, \ldots, T$, excluding account dividends). A global financial portal provided the data (Fusion Media Ltd, n. d.; Finance Yahoo, n. d.). Our sample period is particularly relevant with several major changes occurring during that time. Volkswagen emission problem has caused a decrease in the value of the portfolio (see Figure 1). The time series of the closing prices and the effects of the Volkswagen emission scandal which erupted on 18 September 2015 are shown (Figure 1).

Figure 1 shows the variation of the logarithmic returns in both portfolios and the DAX index. Figure 2 shows the distributions and quantile plots of the returns for EW and MV portfolios in comparison to the DAX respectively. Both portfolios and the DAX index returns do not have normal distribution (Figure 2). The distributions have fat tails, so we have used Student $t$ distribution. Model parameters for Student $t$ distribution were estimated using a maximum likelihood estimation (MLE) method and calibrated in Wolfram Mathematica software using our own procedures (see Table 1). Table 1 displays summary statistics for daily returns for both portfolios and DAX returns. Table 2 reports the results for Student $t$ copulas parameters, as well as the corresponding AIC and BIC values. 
Figure 1: The DAX index and portfolios prices, rebased to 100. The DAX index and portfolios log returns; analyzed period: September, 27, 2010 to June, 28, 2016, daily data

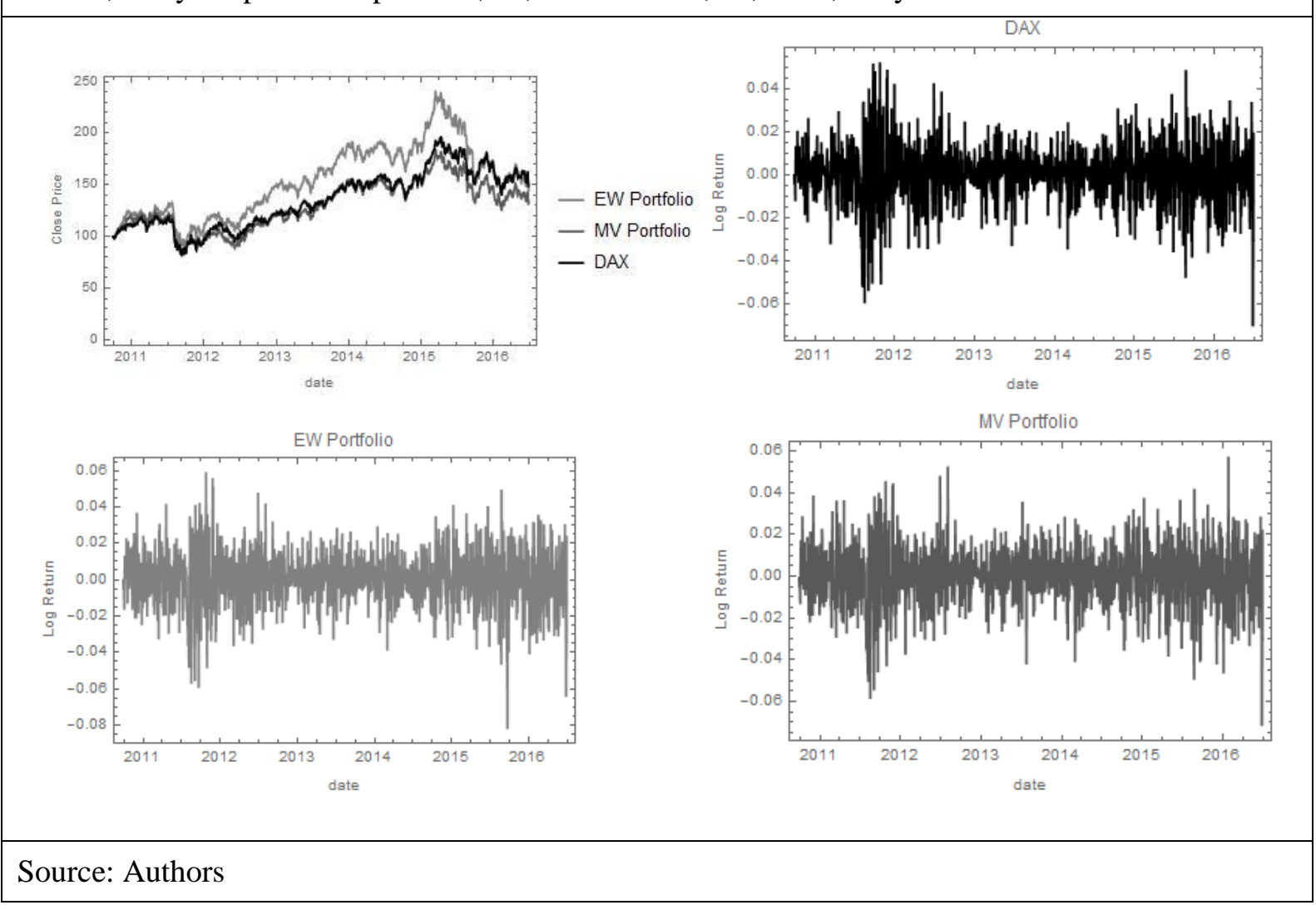

Figure 2: The DAX index and portfolios log returns distributions; analyzed period: September, 27, 2010 to June, 28, 2016, daily data

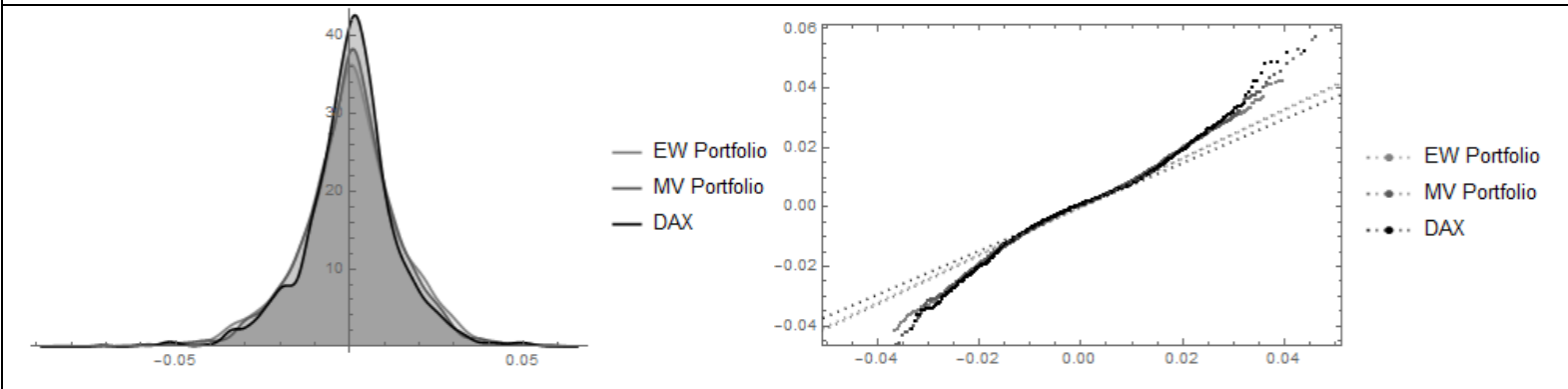

Source: Authors

Table 1: Descriptive Statistics for both portfolios and the DAX returns; analysed period: September, 27, 2010 to June, 28, 2016, daily data

\begin{tabular}{lcrrrrr} 
Portfolio & Mean & $\begin{array}{c}\text { Standard } \\
\text { Deviation }\end{array}$ & $\begin{array}{r}\text { Degree of } \\
\text { Freedom }\end{array}$ & Median & Skewness & Kurtosis \\
\hline $\boldsymbol{E} \boldsymbol{W}$ & 0.00025 & 0.01485 & 5.51225 & 0.00023 & -0.27497 & 4.73780 \\
$\boldsymbol{M} \boldsymbol{V}$ & & & 5.45705 & & & \\
$\boldsymbol{D A} \boldsymbol{X}$ & 0.00018 & 0.01395 & & 0.00043 & -0.23691 & 4.62830 \\
& 0.00027 & 0.01324 & 4.18068 & 0.00081 & -0.28255 & 5.29869
\end{tabular}

Source: Authors 


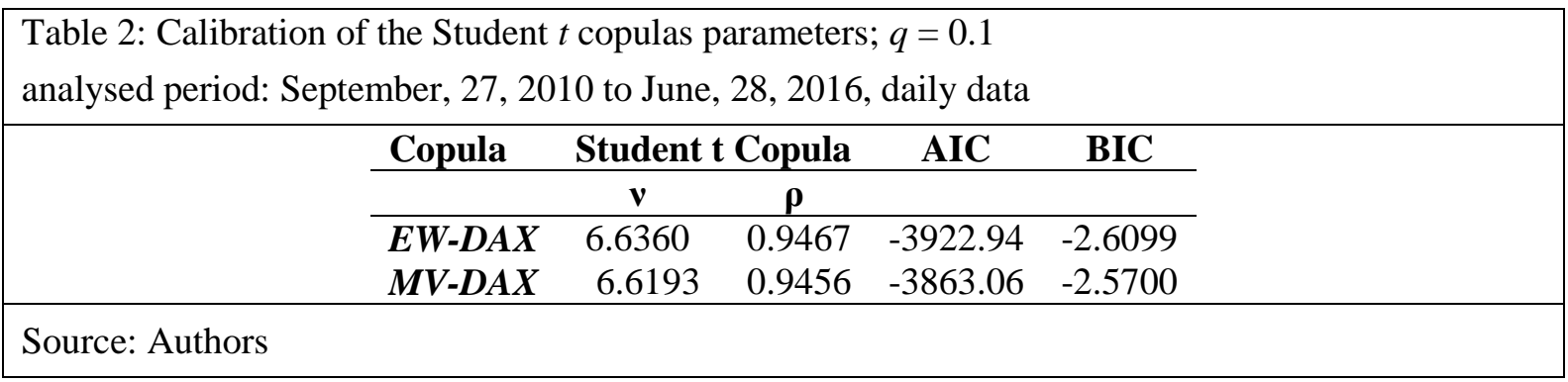

\section{Results and Discussion}

Tables 3-5 compare the optimal hedge ratio computed by the classical approach (OLS), linear quantile regression approach $(\mathrm{QR})$, normal copula quantile regression and Student $t$ copula quantile regression based on $0.1,0.5$ and 0.9 quantile. In both cases, copulas marginal have Student $t$ distribution with parameters as described in Table 1.

Table 3: Estimates of the hedge ratio for quantile $q=0.1$ analysed period: September, 27, 2010 to June, 28, 2016, daily data

\begin{tabular}{lllll} 
Hedge Ratio & OLS & QR & NCQR & SCQR \\
\hline EW Portfolio & 1.1447 & 1.1183 & 1.0673 & 1.0639 \\
MV Portfolio & 1.0752 & 1.0051 & 1.0091 & 1.0038
\end{tabular}

OLSR: Ordinary Least Square Regression, LQR: Linear Quantile Regression, NCQR: Normal Copula Quantile Regression, SCQR: Student $t$ Copula Quantile Regression Source: Authors

Table 4: Estimates of the hedge ratio for the median analysed period: September, 27, 2010 to June, 28, 2016, daily data

\begin{tabular}{lllll} 
Hedge Ratio & OLSR & LQR & NCQR & SCQR \\
\hline EW Portfolio & 1.1447 & 1.1641 & 1.0407 & 1.1023 \\
MV Portfolio & 1.0752 & 1.0134 & 1.0199 & 1.0141
\end{tabular}

OLSR: Ordinary Least Square Regression, LQR: Linear Quantile Regression, NCQR: Normal Copula Quantile Regression, SCQR: Student $t$ Copula Quantile Regression Source: Authors

Table 5: Estimates of the hedge ratio for $q=0.9$

analysed period: September, 27, 2010 to June, 28, 2016, daily data

\begin{tabular}{lllll} 
Hedge Ratio & OLSR & LQR & NCQR & SCQR \\
\hline EW Portfolio & 1.1447 & 1.1713 & 1.0695 & 1.0662 \\
MV Portfolio & 1.0752 & 1.0572 & 1.0115 & 1.0076
\end{tabular}

OLSR: Ordinary Least Square Regression, LQR: Linear Quantile Regression, NCQR: Normal Copula Quantile Regression, SCQR: Student $t$ Copula Quantile Regression Source: Authors 
Our findings suggest that Student $t$ copula quantile regression approach gives the smallest estimations of the hedge ratios for 3 out of 4 cases, while in the fourth case the estimation is very close to the lowest value. Small values of estimations mean we estimate the risks more precisely.

\section{Conclusion}

This paper shows how we can solve the hedge problem using conditional dependence between portfolio returns at a given quantile and the hedging instrument. As a reasonable example, we have analyzed the dependency for quantiles $0.1,0.5$ and 0.9 . It follows from our analysis that we can use non-linear dependency (described by copula function) with returns that are not bivariate normal to obtain more precise results.

\section{References}

Alexander, C. (2008). Market risk analysis. Chichester: John Wiley \& Sons.

Aymen, B. R. \& Mongi, A. (2016). Financial market interdependencies: A quantile regression analysis of volatility spillover. Research in International Business and Finance, 36, 140-157. doi:10.1016/j.ribaf.2015.09.022

Bouyé, E. \& Salomon, M. (2002) Dynamic Copula Quantile Regressions and Tail Area Dynamic Dependence in Forex Markets. Working Paper WP03-01, Financial Econometrics Research Centre, Warwick University. Available from http://www2.warwick.ac.uk/fac/soc/wbs/subjects/finance/research/wpaperseries/wp03-01.pdf

Brooks, Ch., Olan, H. T., \& Persand, G. (2002). The Effect of Asymmetries on Optimal Hedge Ratios. The Journal of Business, Vol. 75, No. 2, April 2002. Available at SSRN: http://ssrn.com/abstract=305879

Fusion Media Ltd. (n. d.). Stock Prices. Retrieved from http://www.investing.com/indices/germany-30

Finance Yahoo (n. d.). Stock Prices. Retrieved from https://finance.yahoo.com

Koenker, R., \& Bassett, G. (1978). Regression quantiles. Econometrica, 46, 33-50

Lien, D., Shrestha, K., \& Wu, J. (2016) Quantile Estimation of the Optimal Hedge Ratio. The Journal of Futures Markets, Vol. 36, No. 2, 194-214, 2015 Wiley Periodicals, Inc. Published online 5 March 2015 in Wiley Online Library

(wileyonlinelibrary.com). DOI: 10.1002/fut.21712 